\title{
ESTUDO DA PROBABILIDADE DE OCORRÊNCIA DE FERRUGEM NO CAFEEIRO CONILON COM O USO DA KRIGAGEM INDICATIVA
}

FONSECA, Abel Souza da ${ }^{1}$

LIMA, Julião Soares de Souza ${ }^{2}$

\begin{abstract}
RESUMO: Este estudo teve por objetivo utilizar a krigagem indicativa para estudar a probabilidade de ocorrência da ferrugem (Hemileia vastatrix) no cafeeiro conilon. Para tal, foram georreferenciados, em uma área de 1,0 ha, 140 pontos (plantas) com auxilio de um GPS topográfico. A avaliação do experimento consistiu na observação da presença ou ausência da doença no terço médio de cada planta do ponto georreferenciado, considerando as plantas com ocorrência da doença igual a 1 (um) e da não ocorrência da doença igual a 0 (zero). A ocorrência da doença na área apresentou dependência espacial, sendo que o mapa construído com o auxilio da krigagem indicativa permite tomar diferentes medidas preventivas em diferentes áreas na lavoura.
\end{abstract}

Palavras chaves: Coffea canephor., Hemileia vastatri., Geoestatística

\section{STUDY THE PROBABILITY OF OCCURRENCE OF RUST IN COFFEE CONILON USING THE INDICATIVE KRIGING}

SUMMARY: This study aimed to use kriging to study the probability of occurrence of rust (Hemileia vastatrix) in coffee conilon. To do so, were georeferenced, in an area of 1,0 ha, 140 points (plants) with the aid of a GPS topographic. The evaluation consisted of the experiment was the observation of the presence or absence of the middle third of each plant geo-referenced point, whereas the plants with disease occurrence equal to 1 (one) and the occurrence of the disease not equal to 0 (zero). The occurrence of the disease in the present spatial dependence, and the map built with the help of kriging allows different take preventive measures in different areas in the field.

Keywords: Coffea canéfora. Hemileia vastatrix. Geostatistics

\section{INTRODUÇÃO}

Um dos grandes problemas enfrentados na produção agrícola na atualidade é a incidência de doenças nas lavouras. Cada cultura possui pragas especificas que são de grande importância, porém, na maioria das vezes, o resultado em casos de infestações é comum nas culturas de interesse agronômico: a perda de produtividade podendo levar a planta a morte. até mesmo a morte das plantas. Para a cultura do café conilon (Coffea canephora), segundo Matiello et al. (1985), a ferrugem é considerada uma das principais doenças de interesse. Esta doença é causada pelo fungo Hemileia vastatrix Berk. \& Br, e se encontra generalizada no país em todas as regiões de cultivo do café conilon (ZAMBOLIM, 2009).

A cafeicultura tem grande importância econômica e social no Brasil e também no estado do Espirito Santo. Sendo este estado o segundo maior produtor de café do país e o maior produtor nacional de café conilon (CONAB, 2012).

\footnotetext{
${ }^{1}$ Engenheiro Agrônomo, Mestrando em Solos e Nutrição de Plantas no Programa de Pós Graduação em Produção Vegetal no Centro de Ciencias Agrarias da Universidade Federal do Espírito Santo. e-mail: abelsouzafonseca@gmail.com;

${ }^{2}$ Engenheiro Agrícola, Prof. do Departamento de Engenharia Rural (CCA-UFES). CP.: 16, CEP: 29500-000, Alegre -ES. e-mail: limajss@yahoo.com.br
} 
Diante da importância da cafeicultura, torna-se necessário um manejo diferenciado que auxilie no aumento da produtividade e no controle da qualidade de grãos, uma exigência cada vez mais comum no mercado. Segundo Zambolim \& Vale (2000), ataques severos do patógeno podem promover perdas de até 50\% de produtividade. A queda precoce das folhas resulta em menor vingamento da florada, menor vingamento dos chumbinhos e seca dos ramos plagiotrópicos comprometendo toda a produção (GREE, 1993; ZAMBOLIM et al., 1997).

Entre as opções de controle, o emprego de fungicidas cúpricos vem sendo utilizado desde a identificação da ferrugem no Brasil (MARIOTTO et al., 1976). Todavia dependendo do regime de chuvas, em determinados anos, torna-se difícil a execução de um programa preventivo de controle da doença, o que permite uma rápida e intensa elevação no índice de ferrugem após um período de chuvas (CHALFOUN et al., 2001). Para Carvalho et al. (2002) uma adubação equilibrada, com realização de desbrotas, podas e plantios menos adensados desfavorecem a incidência da ferrugem.

O conhecimento do modelo de variabilidade espacial da ferrugem do cafeeiro em lavouras contribui para o mapeamento de áreas com maiores e menores incidência da doença. Diante disto é possível realizar os procedimentos necessários para manejar a doença conforme sua distribuição espacial. Segundo Landim (2003), um dos métodos utilizados no estudo da variabilidade da variável é a krigagem, esta corresponde a um processo de estimativa de valores distribuídos no espaço e no tempo, sendo um interpolador exato que leva em consideração todos os valores observados em uma determinada área.

$\mathrm{Na}$ análise espacial a probabilidade de ocorrência de um determinado atributo que está acima ou abaixo de um determinado nível pode ser calculado com o método de krigagem indicativa, como realizado por Zucoloto et al. (2009) no estudo da ocorrência do mal do panamá em bananeira prata anã. A krigagem indicativa consiste basicamente na aplicação da krigagem ordinária para uma variável transformada, ou seja, a variável resultante da aplicação da função não linear $\mathrm{f}(\mathrm{z})=0$ ou 1 (LANDIM, 2003)

Dessa forma, o monitoramento de doenças torna-se uma ferramenta importante no estabelecimento de estratégias e tomada de decisão sobre medidas de manejo. Diante disso, o objetivo deste trabalho foi utilizar a krigagem indicativa para estudar a probabilidade de ocorrência da ferrugem no cafeeiro conilon.

\section{MATERIAL E MÉTODOS}

Os dados utilizados são oriundos de uma área experimental localizada em Cachoeiro de Itapemirim, sul do Espírito Santo. A fazenda pertence ao Instituto Capixaba de Pesquisa, Assistência Técnica e Extensão Rural (INCAPER), e esta localizada nas coordenadas geográficas: $20^{\circ} 45^{`} 17,31^{\prime \prime}$ de Latitude Sul e $41^{\circ} 17^{`} 8,86^{\prime}$ de Longitude Oeste de Greenwich com altitude média na área de 113 metros, conforme Oliveira (2007).

O clima da região é do tipo Cwa, com chuva mal distribuída durante o ano com verão chuvoso e inverno seco de acordo com a classificação de Koppen. A temperatura média do mês mais frio é inferior $20^{\circ} \mathrm{C}$ e do mês mais quente superior a $27^{\circ} \mathrm{C}(\mathrm{COSTA}, 2011)$. Os dados climáticos foram fornecidos pela estação metereológica do INCAPER, localizada na área de estudo.

A espécie de café utilizada no trabalho foi a Coffea canephora Pierre, var. Robusta Tropical (Emcaper 8151), sendo que a cultura se encontra em espaçamento tradicional de 2,9 m entre linhas e $0,9 \mathrm{~m}$ entre plantas. O manejo anual da adubação foi feito com parcelamento de nitrogênio $(\mathrm{N})$, potássio $(\mathrm{K})$ e fósforo $(\mathrm{P})$, sendo empregados 130 gramas por planta do formulado 20-00-20 e uma aplicação de 80 gramas por planta de super simples (SS). A poda de produção foi realizada anualmente, após cada colheita buscando manter, aproximadamente, 12.000 hastes por hectare na área experimental.

Dentro da área experimental foram georreferenciados, em uma área de 1,0 ha, 140 pontos (plantas), que são representativos de toda a área em estudo, com auxílio de um GPS topográfico, modelo GTR-1. A 
avaliação do experimento foi no inicio de junho de 2011, e consistiu na observação da presença ou ausência da ferrugem (Hemileia vastatrix) no terço médio de cada planta do ponto georreferenciado. Para isso foi escolhido aleatoriamente um ramo plagiotrópico em cada quadrante da planta, totalizando quatro ramos. Em seguida contou-se o número total de folhas em cada ramo e observado se tinha ou não tinha incidência de ferrugem. Ocorrendo a incidência de ferrugem em um dos ramos, a planta foi classificada como planta contaminada pelo fungo.

A probabilidade de ocorrência da ferrugem na área foi avaliada pelo método de interpolação krigagem indicativa, que é um processo básico de interpolação. Para atingir o objetivo, o primeiro passo foi considerar as plantas com ocorrência da doença igual a 1 (um) e da não ocorrência da doença igual a 0 (zero), conforme Landim (2003) na análise estatística de dados geológicos.

Assumida a hipótese de estacionaridade, ou seja, existência da semivariância através de um modelo matemático, cuja presença ou ausência da doença foi submetido à análise geoestatística, no intuito de verificar a probabilidade de ocorrência por meio do ajuste do semivariograma, que é definido pela Equação 1:

$$
V_{\mathrm{i}}\left(\mathrm{h}, \mathrm{V}_{\mathrm{c}}\right)=\frac{1}{2 \mathrm{~N}_{\mathrm{h}}} \sum_{\mathrm{i}=1}^{\mathrm{N}_{\mathrm{h}}}\left[\mathrm{i}\left(\mathrm{x}+\mathrm{h}, \mathrm{V}_{\mathrm{C}}\right)-\mathrm{i}\left(\mathrm{x}, \mathrm{V}_{\mathrm{C}}\right)\right]^{2}
$$

Onde:

$\mathrm{h}=$ passo (lag) básico;

$\mathrm{V}_{\mathrm{c}}=$ nível de corte;

$\mathrm{N}=$ número de pares.

O software GS+ (ROBERTSON, 1998) utiliza a metodologia dos mínimos quadrados para ajustes dos modelos teóricos aos semivariogramas experimentais, determinando os parâmetros: efeito pepita (C0), patamar $(\mathrm{C} 0+\mathrm{C})$ e o alcance de dependência espacial (a). Os critérios para ajuste e seleção do melhor modelo foram o coeficiente de determinação $\left(\mathrm{R}^{2}\right)$, soma de quadrados de resíduos (SQR) e o coeficiente de correlação da validação cruzada. Deste modo, o índice de dependência espacial (IDE) foi calculado através da relação [C/(C0 $+\mathrm{C}$ ) $* 100$, classificado segundo Zimback (2001), assumindo, assim, os seguintes intervalos: dependência espacial baixa para IDE $\leq 25 \%$, moderada para $25 \%<$ IDE $<75 \%$ e forte para IDE $\geq 75 \%$.

Comprovado a dependência espacial, foi gerada uma malha interpolada da probabilidade de ocorrência da ferrugem, e com auxilio do software Surfer, Golden software (2002), gerando o mapa temático através de uma planilha de dados formato (DAT) já interpolados pelo GS+.

\section{RESULTADOS E DISCUSSÃO}

Após a transformação dos dados procedeu-se a analise geoestatística, apresentada na Tabela 1, sendo que o modelo de semivariograma que melhor se ajustou foi o esférico, com um alcance (a) de 5 metros, ou seja, pontos coletados com distância maiores que este valor são considerados independentes e, para sua análise, pode-se utilizar a estatística clássica (VIEIRA, 2000).

Tabela 1. Modelo e parâmetros estimados dos semivariogramas experimentais para os dados de ferrugem em cafeeiro conilon

\begin{tabular}{ccccccc}
\hline Variável & Modelo & $\begin{array}{c}\mathrm{a} \\
(\mathrm{m})\end{array}$ & $\mathrm{C}_{0}$ & $\mathrm{C}_{0}+\mathrm{C}$ & $\begin{array}{c}\mathrm{IDE} \\
(\%)\end{array}$ & $\begin{array}{c}\mathrm{R}^{2} \\
(\%)\end{array}$ \\
\hline Ferrugem & Esf. & 5,0 & 0,06 & 0,22 & 70,0 & 80,0
\end{tabular}

Esf- modelo esférico; a - alcance; $\mathrm{C}_{0}$ - efeito pepita; $\mathrm{C}_{0}+\mathrm{C}$ - patamar; IDE - índice de dependência espacial $\left[\mathrm{C} /\left(\mathrm{C}_{0}\right.\right.$ $+\mathrm{C})$ ]; a - alcance (m) e $\mathrm{R}^{2}$ - coeficiente de determinação do modelo do semivariograma. 
O efeito pepita representa a variabilidade não explicada e segundo Lima et al. (2006), quanto menor a proporção do efeito pepita em relação ao patamar do semivariograma, maior será a dependência espacial apresentada pelo atributo, que neste experimento foi considerada moderada segundo a classificação de Zimback (2001).

A doença tem uma tendência de maior ocorrência na lateral esquerda da área estudada, como mostrado nas Figuras 1 e 2. Com essas informações é possível assumir que esta região necessita de uma maior atenção nas tomadas de medidas preventivas de manejo. Isto irá impedir que a área se torne fonte de inoculo do patógeno para outras regiões com menor probabilidade de ataque. Segundo Ferrão (2007), os esporos podem ser disseminados através do vento, insetos, chuva, animais e outros. Para Zambolim et al. (2000) possíveis consequências negativas da doença na área, como a diminuição da produção devem ser minimizadas.

Figura 1. Mapa temático de isolinhas da probabilidade de ocorrência da ferrugem no cafeeiro variando de 0 (isenta da doença) a um ( presença da doença)

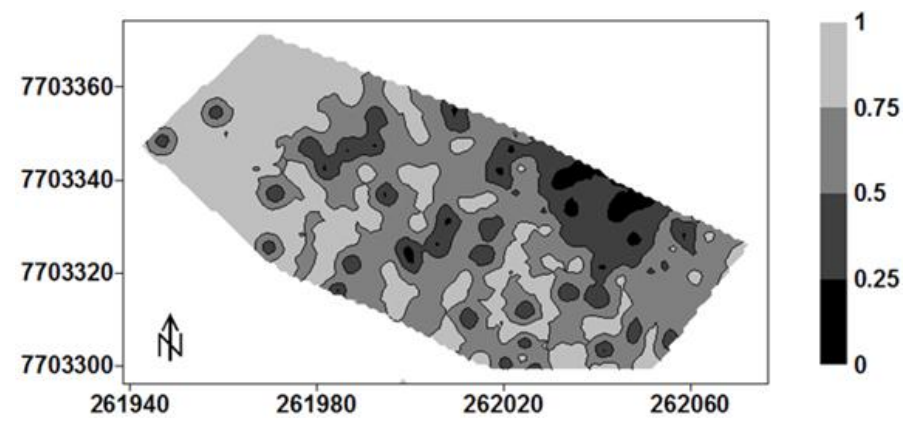

Figura 2. Mapa temático de isolinhas da probabilidade de ocorrência da ferrugem no cafeeiro variando de 0 a 0,75 e de 0,75 a 1

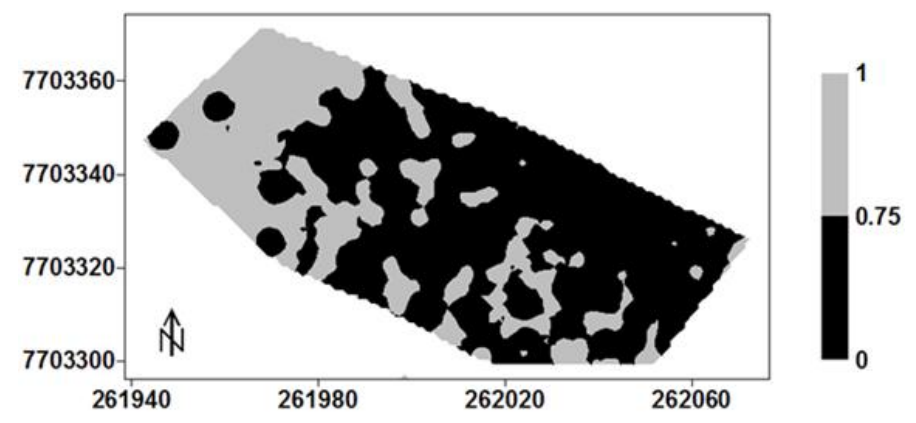

A Figura 2 representa a probabilidade de ocorrência de $0 \%$ a $75 \%$ e $75 \%$ a $100 \%$, com maiores detalhes da área. A área mais clara possui de 75\% a $100 \%$ de chance da doença causar danos à cultura, reduzindo a sua produtividade, sendo que a atenção do produtor deve estar voltada para a mesma, sem se esquecer das áreas restante, que possuem menor probabilidade de ocorrência. Essa área também possui importância quanto ao manejo para uma boa produtividade e a obtenção de produtos de melhor qualidade.

O uso dos mapas construídos pela krigagem indicativa permite antecipar ações preventivas sobre a doença na área em estudo, diminuindo o uso de pesticidas, com aplicação localizada ao invés de uma aplicação homogênea, com consequente redução dos custos de produção e de danos ao meio ambiente e ao homem.

$\mathrm{O}$ manejo da ferrugem envolve o principio da proteção e terapia preventiva com os fungicidas de contato, sendo os cúpricos os mais efetivos, ou emprego de fungicidas sistêmicos via solo e, ou pulverização 
foliar (ZAMBOLIM et al., 1997). Informações preventivas auxiliarão sobre quando iniciar as pulverizações, a que intervalos elas devem ser feitas e a frequência que devem ser realizadas (CAMPBELL; MADDEN, 1990).

O equilíbrio nutricional do cafeeiro é um meio de prevenção de doenças. Plantas desbalanceadas nutricionalmente são mais susceptíveis a doenças (MARSCHNER, 1995). Segundo o mesmo autor, a nutrição mineral balanceada funciona como barreira física, evitando a penetração das hifas, através da cutícula espessa e auxilia na formação de compostos fenólicos, com propriedades fungistáticas. Portanto, a visualização da área afetada e antecipação de medidas de manejo, tais como: uma análise mais criteriosa do solo e da planta buscando corrigir algum desequilíbrio nutricional do cafeeiro será importante para prevenção da doença.

Fatores climáticos influenciam no desenvolvimento da ferrugem, condicionando a distribuição da doença, assim como sua incidência e severidade (FERRÃO, 2007). Dentre os fatores que governam o desenvolvimento do patógeno, são ambientes sombrios e espaçamento mais fechado (ZAMBOLIM et al., 1985). Desta forma, a realização de desbrotas e podas para evitar o fechamento da lavoura, favorecendo a entrada da luz solar, são procedimentos que reduzem o sombreamento da lavoura, desfavorecendo a incidência da ferrugem (CARVALHO et al., 2002). Sendo este manejo realizado de forma mais intensa nas zonas com maior probabilidade de ocorrência da ferrugem.

\section{CONCLUSÃO}

- A ocorrência da doença na área apresenta dependência espacial, com ajuste ao modelo esférico.

- A metodologia utilizada permite tomar medidas preventivas localizadas quanto ao ataque do patógeno causador da ferrugem.

\section{REFERÊNCIAS}

CAMPBELL, C. L.; MADDEN, L. V. Introduction to Plant Disease Epidemiology. New York: John Willey \& Sons, 1990. 532p.

CARVALHO, V.L.; CUNHA, R.L.; CHALFOUN, S.M. Manejo ecológico das principais doenças do cafeeiro. Informe Agropecuário, Belo Horizonte, v.23, n. 214/215, p.101-114., 2002.

CHALFOUN, S. M.; CARVALHO, V. L. DE; PEREIRA, M. C. Efeito de alterações climáticas sobre o progresso da ferrugem (Hemileia vastratrix Berk. \& Br.) do cafeeiro (Coffea arabica L.). Ciência e

Agrotecnologia, Lavras, v. 25, n. 5, p. 1248-1252, 2001.

CONAB - COMPANHIA NACIONAL DE ABASTECIMENTO. Acompanhamento da Safra Brasileira Quarta Estimativa, Dezembro/2012. Online. Disponível em:

<http://www.conab.gov.br/OlalaCMS/uploads/arquivos/12_12_20_16_01_51_boletimcafe_dezembro_2012.pdf

$>$ Acesso em 01 fev. 2013.

COSTA, F. P. Uso da geoestatística e da lógica fuzzy no estudo da variabilidade espacial e temporal da produtividade e da fertilidade do solo em café conilon. 2011. 75f. Dissertação Mestrado - Universidade Federal do Espírito Santo, Alegre.

GOLDEN SOFTWARE. Surfer: Surface mapping system (version 8.0). Surface mapping system. Colorado: Golden, Inc, Software, 1993-2002. 1 CD-ROM.

GREE, G. Epidemiology of coffee leaf rust in the Eastern Highlands. Newsletter - Coffee Research Institute, v.2, p.16-20. 1993.

LANDIM, P. M. B. Análise estatística de dados geológicos. 2.ed. São Paulo. UNESP, 2003. 253p. 
MARIOTTO, P. R.et al. Estudos sobre o controle químico da ferrugem do cafeeiro (Hemileia vastatrix Berk \& Br.) e seus efeitos na produção, nas condições do estado de São Paulo. , v. 45, n. 910, p. 165-174, 1976.

MARSCHNER, H. Mineral Nutrition of Higher Plants. 2ed. San Diego: Academic Press, 1995. 889 p.

MATIELLO, J.B. et al. Cultura de Café no Brasil: manual de recomendações. 5 ed. Rio Janeiro: IBCGERCA, 1985. $580 \mathrm{p}$.

OLIVEIRA, R. B. Mapeamento e Correlação de Atributos do Solo e de Plantas de Café Conilon para fins de Agricultura de Precisão. 2007. 129 f. Dissertação Mestrado - Universidade Federal do Espírito Santo, Alegre.

FERRÃO, R. G. et al. Técnicas de produção com variedades melhoradas 3. ed. Revista Café Conilon. VitóriaES: Incaper. p. 60., 2007.

VIEIRA, S. R. Uso de geoestatística em estudos de variabilidade espacial de propriedades do solo. In: NOVAIS, R. F.; ALVAREZ, V. H. V.; SCHAEFER, C. E. G. R. (Org.). Tópicos em Ciência do Solo. Viçosa: Sociedade Brasileira de Ciência do Solo, 2000, v. 1, p. 1-54.

ROBERTSON, G.P. GS+: geostatistics for the environmental sciences. Plainwell: Gamma Design Software, 2008.

ZAMBOLIM, L.et al. Café (Coffea arabica L.). Controle de doenças causadas por fungos, bactérias e vírus. In: VALE, F.X.R.; ZAMBOLIM, L. (Eds.) Controle de doenças de plantas. Viçosa: Suprema Gráfica e Editora, 1997. p. 83-180.

ZAMBOLIM, L.; MARTINS, M.C. DEL P.; CHAVES, G.M. Café. Informe Agropecuário. Belo Horizonte, v.11, n.131, p.64-75, 1985.

ZAMBOLIM, L.; VALE, F.X.R. Perdas na produtividade e qualidade do cafeeiro causadas por doenças bióticas e abióticas. In: ZAMBOLIM, L. Café: produtividade, qualidade e sustentabilidade. Viçosa: UFV, 2000. p. $239-261$.

ZAMBOLIN, L. Tecnologias para produção do Café Conilon. Viçosa: UFV, 2009. 360p

ZIMBACK, C. R. L. Análise espacial de atributos químicos de solos para fins de mapeamento da fertilidade do solo. 2001. 114 f. Tese (Livre Docência). Universidade Estadual Paulista., Botucatu.

ZUCOLOTO, M.; LIMA, J. S. S.; COELHO, R. I.; Uso da geoestatística na probabilidade de ocorrência do mal-do-panamá em bananeira prata anã. Revista Facultad Nacional de Agronomía Medellín, v. 62, n.1, p.4793-4796, 2009. 